\title{
p53 Expression in Triple Negative Breast Carcinomas: Evidence of Age-Related and Racial Differences*
}

\author{
Simone Davion, Megan Sullivan, Stephen Rohan, Kalliopi P. Siziopikou ${ }^{\#}$ \\ Department of Pathology, Breast Pathology Section, Northwestern University Feinberg School of Medicine, Chicago, USA. \\ Email: "p-siziopikou@northwestern.edu
}

Received May 31 ${ }^{\text {st }}$ 2012; revised June 30 ${ }^{\text {th }}$, 2012; accepted July $10^{\text {th }}, 2012$

\begin{abstract}
Triple negative breast carcinomas (TNBC), are defined by the absence of estrogen receptor (ER), progesterone receptor (PR) and human epidermal growth factor receptor 2 (HER2) expression. The majority of TNBC are "basal-like", a group originally defined by studies of mRNA gene expression profiles, but increasingly defined in the clinic by using surrogate markers such as CK 5/6. However, not all TNBC are basal-like. It is postulated that these subcategories of TNBC have distinct underlying biologies that drive their ultimate behavior, and response to treatment, with important implications for designing appropriate targeted therapies. In this study we report that within our cohort of 197 TNBC, distinct groups were identified that varied by CK 5/6 and p53 expression based on age and race. We propose that awareness of CK5/6 and p53 expression in younger and AA TNBC patients may facilitate identifying patients with unique tumor subtypes and may lead to better use of targeted therapies in this group of aggressive breast cancers.
\end{abstract}

Keywords: Triple Negative Breast Cancer; p53; Race; Age

\section{Introduction}

Triple negative breast carcinomas (TNBC) represent 10\% - $17 \%$ of breast carcinomas and are defined immunohistochemically by lack of expression for estrogen receptor (ER), progesterone receptor (PR) and human epidermal growth factor receptor 2 (HER2) [1]. TNBC are a heterogeneous group of tumors with different pathologic characteristics, molecular alterations, clinical features and biologic behavior. Basal-like breast tumors were so named because they express many of the genes found in normal breast myoepithelial cells including cytokeratin filaments 5, 14, and 17. Many of these "basal-like" breast cancers also have low expression of ER as well as genes co-expressed with ER. Basal-like tumors represent between $50 \%-75 \%$ of TNBC [2-4]. However, breast cancers from each intrinsic molecular subtype, including luminal A, luminal B, Her-2-enriched and claudin-low, have also been identified within the triple negative group. Triple negative tumors are most often high grade infiltrating ductal carcinoma of no special type, exhibiting rapid growth, often declaring themselves before mammographic screening has begun in young women or in

\footnotetext{
*The authors have no financial disclosures, conflicts of interest or acknowledgements to report.

This study was conducted after approval by the Institutional Review Board (IRB) of Northwestern University and complies with the US policies on ethical consent.

${ }^{\#}$ Corresponding author.
}

between mammograms (interval cancers) in older women [1]. TNBC are not amenable to targeted therapies with either hormone antagonists, such as tamoxifen, or antibodies targeting HER2 such as trastuzamab. These tumors often respond initially to classes of more indiscriminate chemotherapies but have a high likelihood of recurring or progressing [5]. Recently the use of PARP-1 inhibitors in a subgroup of basal-like TNBC has been entertained and thus accurate subclassification of TNBC into basal-like and other subtypes is becoming more clinically relevant [6].

The p53 protein exercises control over cell cycle progression via its role as a transcription factor that regulates expression of a large number of genes which govern cell cycle arrest and progression. Over half of human cancers acquire p53 mutations during malignant transformation resulting from either loss of function or gain of function mutations. Gain of function mutations in p53 result from a change in a single amino acid in the DNA binding domain, leading to inactivity, increased stability, and expression of high levels of p53 protein detectable by immunohistochemistry [7]. Immunohistochemical staining for the p53 protein, performed on tissue sections, is a proxy for gain of function mutations in p53 that result in inactivity.

Mutations in the p53 gene have been found to be more common in basal-like cancers than other breast cancer subtypes and may interact with retinoblastoma protein RB1 in claudin-low TNBC [8-10]. p53 gain of function 
mutations and consequent protein expression, within the group of triple negative breast cancers, has been found to meaningfully stratify this heterogeneous group of breast cancers into subtypes with better or worse prognosis, and differing response to chemotherapy $[11,12]$.

Demographically the basal subtype of TNBC has been demonstrated to more commonly affect young African American (AA) women. Hypotheses have been put forward that these biologic differences may explain the lower incidence but higher mortality of breast cancer in this cohort of breast cancer patients [4,13-15].

In this study we evaluated the role of p53, a mediator of cellular response to DNA damage, in a high risk group of breast cancers with limited therapeutic options, as it relates to race and age in a cohort of patients diagnosed at a single academic medical center between 2003-2010.

\section{Materials and Methods}

This study was conducted after approval of the institutional review board at Northwestern University. The electronic pathology laboratory information system was searched for cases of breast carcinoma diagnosed between 2003-2010. Inclusion criteria were carcinomas with documented negativity for ER, PR, and HER2, and the availability of paraffin blocks with representative tumor and sufficient tissue for immunohistochemical studies. To be defined as a triple negative carcinoma, the tumor had to show $<1 \%$ nuclear positivity with estrogen receptor and progesterone receptor and be negative for HER2 by either immunohistochemistry (score 0 or $1+$ ) or FISH analysis. Pathologic tumor characteristics included histological type, tumor grade, tumor size, presence of lymphatic/vascular invasion (LVI), lymph node status and p53 expression. At our institution p53 expression by IHC is performed routinely on all newly diagnosed invasive carcinomas of the breast. Cases were considered to be positive for p53 when $>10 \%$ of tumor cells demonstrated nuclear staining.

Electronic medical records were reviewed to determine patient demographics including age and race. Race was determined either by self-identification at the time of initial visit, or designated by the clinician at the initial intake visit. For purposes of analysis race was categorized as Caucasian, African American, Asian, Hispanic or Other.

\subsection{Tissue Microarray Construction}

Ninety-nine cases of TNBC with more than one tumor block available were used to construct tissue microarrays (TMAs). The TMAs were constructed from the formalin fixed paraffin embedded tissue blocks. For each patient, three separate $0.2 \mathrm{~cm}$ tumor cores were included to account for tumor heterogeneity. Embedded within each
TMA were additional cores of positive and negative control tissue.

\subsection{Immunohistochemical Studies}

Immunohistochemical studies were carried out on the TMAs with antibodies to cytokeratin 5/6 (DAKO D5/ 16b4). Staining for CK 5/6 was carried out at 1:25 solution after retrieval with mild cell conditioning solution 1 . Positive control tissue both embedded within the TMA and on separate slides was evaluated to ensure that the results were interpretable. CK 5/6 was determined to be positive with any degree of positive cytoplasmic staining in accordance with the literature [16].

\section{Statistical Analysis}

The data were analyzed with cross tabulations and the chi-squared test was used as appropriate. p-values $<0.05$ were considered statistically significant.

\section{Results}

\subsection{Clinicopathologic Data}

One hundred and ninety-seven cases of TNBC were retrieved from the electronic laboratory information system. Patient age at diagnosis ranged from 29 - 93 (mean age: 54). Of the 197 TNBC $45 / 197$ (22.8\%) were AA, 142/197 (72.0\%) were Caucasian, 4/197 (2\%) were Hispanic. 2/197 (1\%) were Asian, and 4/197 (2\%) patients did not have racial information recorded in the electronic medical record.

TNBC were almost exclusively infiltrating ductal carcinomas of no special type (181/197, 92.3\%). The remaining cases were either invasive lobular carcinoma 2/19 (1\%), metaplastic carcinoma 13/197 (6.6\%), or adenoid cystic carcinoma $1 / 197(\mathrm{p}=0.05 \%)$ Using the modified Bloom Richardson grading system, the majority of the ductal and lobular carcinomas were grade 3 tumors (150/183, 82\%), and the remaining were grade 2 (33/183, 18\%). None of the tumors were histologic grade 1 (Table 1).

Table 1. Histologic and breast marker characteristics of the patient population.

Tumor characteristics $(\mathrm{n}=197)$

\begin{tabular}{rc}
\hline ER negative & $100 \%$ \\
PR negative & $100 \%$ \\
HER-2 negative & $100 \%$ \\
Ductal histology & $92.3 \%$ \\
High histologic grade (III) & $82.7 \%$ \\
p53 expression & $54.7 \%$ \\
\hline
\end{tabular}




\subsection{Correlation between $\mathrm{p} 53$ Expression and Race}

Overall 67 patients who had their race recorded in the electronic medical record (18 AA and 49 Caucasian patients) had their p53 status available. Amongst TNBC p53 expression was associated with African-American (AA) race. Specifically more than three quarters 14/18 (77.7\%) of AA patients with TNBC expressed p53 compared to just over half 28/49 (57.1\%) of Caucasians (p < 0.01) (Table 2). p53 status was not associated with the tumor grade, tumor size, presence of LVI nor lymph node status.

\subsection{Correlation between Basal Phenotype and p53 Expression}

The basal phenotype of TNBC as defined by cytokeratin $5 / 6$ expression was then correlated with expression of p53. 83 cases had both p53 and CK 5/6 data available. p53 was expressed in a higher percentage of basal phenotype (CK 5/6 positive) cases ( $29 / 48,60.4 \%$ ) compared to just over half of the CK 5/6 negative cases (18/35, 51.4\%) (Table 3).

\subsection{Correlation between Age and CK 5/6-Positive (Basal)/p53-Positive Phenotype}

Of interest, of the 83 cases of TNBC that had both p53 and CK 5/6 status available, twice as many (50\%) of the TNBC patients younger than 50 expressed the basal/p53 positive phenotype compared with only $23.4 \%$ of the TNBC patients older than $50(\mathrm{p}<0.01)$, independent of race (Table 4 and Figure 1).

Table 2. Correlation between race and p53 expression.

\begin{tabular}{cccc}
\hline \multicolumn{4}{c}{ p53 Expression } \\
\hline Race & Positive & Negative & Total \\
African-American & $14(77.7 \%)$ & $4(22.3 \%)$ & 18 \\
Caucasian & $28(57.1 \%)$ & $21(42.8 \%)$ & 49 \\
Total & 42 & 25 & 67 \\
\hline
\end{tabular}

$\mathrm{p}<0.01$.

Table 3. Correlation between basal phenotype and p53 expression.

\begin{tabular}{cccc}
\hline \multicolumn{4}{c}{ p53 Expression } \\
\hline CK5/6 Expression & Positive & Negative & Total \\
Positive & $29(60.4 \%)$ & $19(39.5 \%)$ & 48 \\
Negative & $18(51.4 \%)$ & $17(48.5 \%)$ & 35 \\
Total & 47 & 36 & 83 \\
\hline
\end{tabular}

Table 4. Correlation between age and CK 5/6-positive (basal)/p53-positive phenotype.

\begin{tabular}{cccc}
\hline Age & Phenotype Present & Phenotype Absent & Total \\
\hline$<50$ yo & $18(50 \%)$ & $18(50 \%)$ & 36 \\
$>50$ yo & $11(23.4 \%)$ & $36(76.6)$ & 47 \\
Total & 29 & 54 & 83 \\
\hline$<<0.01$ & & &
\end{tabular}

\section{Discussion}

Our study of a cohort of TNBC diagnosed at a single academic medical center between 2003-2010, shows that p53 expression is seen in over half of TNBC and that TNBC patients younger than 50 years of age are twice as likely to have p53 expressing TNBC of the basal-like phenotype than patients older than 50 years of age, regardless of race. Results from our cohort also showed that the basal phenotype/p53 positive TNBC is more common in African Americans compared to Caucasians.

Our results are consistent with the Carolina Breast Cancer study and others $[4,15,17,18]$. Awareness of these associations between intrinsic biologic differences and different tumor phenotypes, as well as the prevalence of different tumor phenotypes in different ethnic groups is becoming more important for successful application of individualized targeted therapies.

It is well documented that African American women with breast cancer have a poorer prognosis than Caucasians. The relative risk of death for African American women with breast cancer compared to Caucasian patients is between 1.10 and 1.22, and while the overall incidence rates for breast cancer amongst African American is lower than Caucasian women, 128.95 compared to 114.12 per 100,000 , the 5 year survival rate for African Americans is lower than Caucasians, 91.4\% versus $77.4 \%[19,20]$.

Many explanations for this disparity have been proffered including variation in stage at diagnosis, treatment, and access to care, but even after controlling for such variables African American women still have a higher mortality rate from breast cancer than Caucasians [21]. Several groups have reported that the biology of breast cancers in African Americans is different, with African American women more commonly having triple negative breast cancers than their Caucasian counterparts $[14,15$, 21].

Similarly premenopausal women with breast cancer have lower 5 year survival rates than post-menopausal women with breast cancer, $83 \%$ versus $90 \%[22,23]$. The biology of breast cancers in younger women appears to be distinct based on histopathologic, immunohistochemical, and mRNA gene expression analysis. Breast 


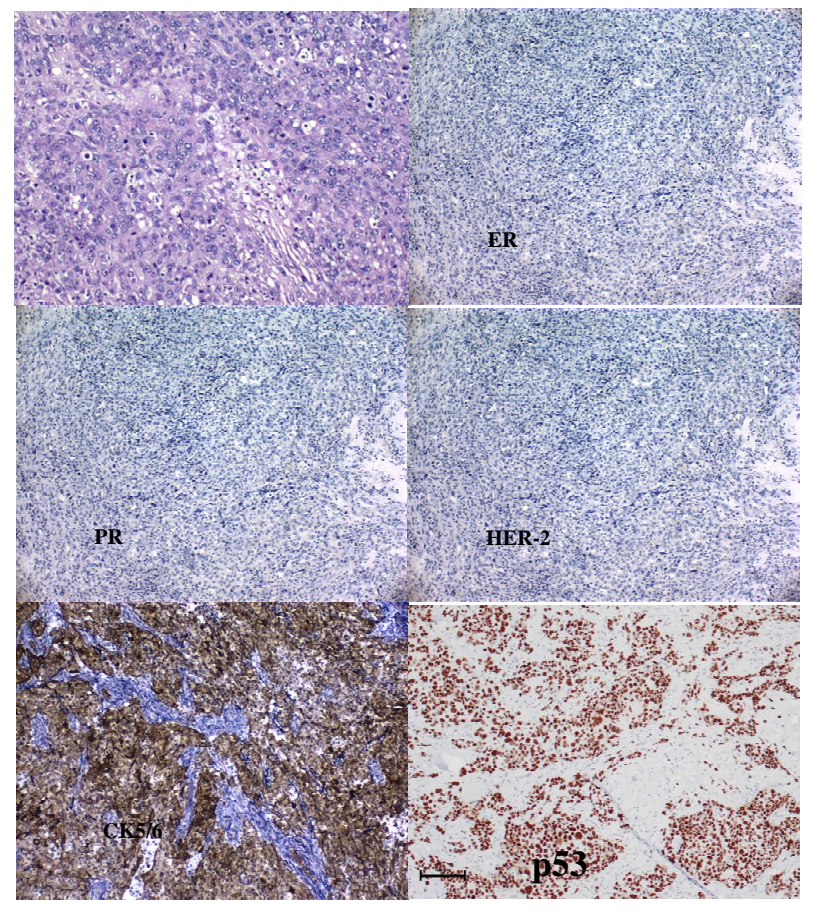

Figure 1. Infiltrating ductal carcinoma grade III/III, ERnegative/PR-negative/HER2-negative exhibiting strong CK $5 / 6$ positivity and high p53 expression.

cancers in younger women is more often of higher grade, larger size, with more frequent lymph node positivity. Gene expression profiles of breast cancer in younger women demonstrates that these cancers have lower levels of $\mathrm{ER} \alpha$ and progesterone receptor expression, with higher expression of gene subsets related to stem cells and BRCA1 $[24,25]$. Tumors in younger women have also been shown to be less responsive to treatment [22, 23].

The p53 tumor suppressor gene has long been recognized as an important regulator of the normal cell cycle and mutations in p53 are a major factor in malignant transformation and tumor progression. Over half of human cancers acquire p53 mutations during malignant transformation resulting from either loss of function or gain of function mutations. p53 mutations have been shown to be prognostic in breast cancer. Patients with p53 mutated breast cancers have worse disease free survival, independent of other risk factors and p53 mutations have been hypothesized to play a role in resistance to certain chemotherapies [25-27]. Emerging studies are attempting to elucidate the correlation between p53 mutations and mutations in retinoblastoma protein (RB1) in TNBC and the claudin-low tumors. The claudin-low tumors are associated with tumor cells that may possess stem cell qualities such as the ability for self-renewal and resistance to chemotherapy $[10,28]$.

At this time, routine use of molecular markers such as p53 and CK 5/6 in defining subgroups of breast cancer patients, although occasionally reported, is not established pathology practice. However, as the association between p53 mutations, basal subtype, BRCA1 mutations, and the emerging sensitivity of BRCA1 deficient tumors to PARP inhibitors is beginning to be established, and the importance of p53 is once again taking center stage [2].

Furthermore gene microarray analysis has shown that breast carcinomas can be further classified by their p53 status and this "p53 signature" predicts mutation status and patient survival in different patient subsets independent of other risk factors [27]. Of interest, combined mutations in retinoblastoma gene RB1 and p53 in mammary stem cell progenitors is reported to induce epithelial to mesenchymal transition (EMT) in animal models [10]. In triple negative mammary tumors in mice the EMT-type tumor and a subset of basal phenotype tumors, are reported to express mutant forms of p53, suggesting that p53 plays a major role in dictating tumor subtype after RB inactivation in triple negative breast cancers [29]. It is tempting to speculate that these pathways are likely to also play a major role in the pathogenesis of TNBC in humans and are possibly important in determining response to therapy and overall prognosis.

Finally, as therapies targeting the specific subtypes of TNBC are introduced into wider clinical use, expansion of the routine panel of immunohistochemical tumor markers performed on invasive breast cancers to include p53 and basal-type markers may be necessary. Awareness of the increased likelihood of finding these tumors in TNBC patients younger than 50 , as well as AA patients may ultimately lead to successful individualized regimens for these difficult to treat subtypes of breast carcinomas.

\section{REFERENCES}

[1] W. D. Foulkes, I. E. Smith and J. S. Reis-Filho, "Triple-Negative Breast Cancer,” The New England Journal of Medicine, Vol. 363, No. 20, 2010, pp. 1938-1948. doi:10.1056/NEJMra1001389

[2] C. M. Perou, "Molecular Stratification of Triple-Negative Breast Cancers,” Oncologist, Vol. 16, Suppl. 1, 2011, pp. 61-70. doi:10.1634/theoncologist.2011-S1-61

[3] C. M. Perou, T. Sorlie, M. B. Eisen, et al., "Molecular Portraits of Human Breast Tumours,” Nature, Vol. 406, No. 6797, 2000, pp. 747-752. doi:10.1038/35021093

[4] K. A. Dookeran, J. J. Dignam, K. Ferrer, M. Sekosan, W. McCaskill-Stevens and S. Gehlert, "p53 as a Marker of Prognosis in African-American Women with Breast Cancer,” Annals of Surgical Oncology, Vol. 17, No. 5, 2010, pp. 1398-1405. doi:10.1245/s10434-009-0889-3

[5] C. M. Perou, "Molecular Stratification of Triple-Negative Breast Cancers,” Oncologist, Vol. 15, Suppl. 5, 2010, pp. 
39-48. doi:10.1634/theoncologist.2010-S5-39

[6] E. A. Comen and M. Robson, "Poly (ADP-Ribose) Polymerase Inhibitors in Triple-Negative Breast Cancer," Cancer, Vol. 16, No. 1, 2010, pp. 48-52. doi:10.1097/PPO.0b013e3181cf01eb

[7] K. H. Vousden and D. P. Lane, "p53 in Health and Disease,” Nature Reviews, Vol. 8, No. 4, 2007, pp. 275-283. doi:10.1038/nrm2147

[8] N. Turner, A. Tutt and A. Ashworth, "Hallmarks of 'BRCAness' in Sporadic Cancers," Nature Reviews, Vol. 4, No. 10, 2004, pp. 814-819. doi:10.1038/nrc1457

[9] T. Sorlie, C. M. Perou, R. Tibshirani, et al., "Gene Expression Patterns of Breast Carcinomas Distinguish Tumor Subclasses with Clinical Implications,” Proceedings of the National Academy of Sciences of the United States of America, Vol. 98, No. 19, 2001, pp. 10869-10874. doi:10.1073/pnas.191367098

[10] Z. Jiang, R. Jones, J. C. Liu, et al., "RB1 and p53 at the Crossroad of EMT and Triple-Negative Breast Cancer," Cell Cycle, Vol. 10, No. 10, 2011, pp. 1563-1570. doi:10.4161/cc.10.10.15703

[11] E. Biganzoli, D. Coradini, F. Ambrogi, et al., "p53 Status Identifies Two Subgroups of Triple-Negative Breast Cancers with Distinct Biological Features," Japanese Journal of Clinical Oncology, Vol. 41, No. 2, 2011, pp. 172-179. doi:10.1093/jjco/hyq227

[12] B. Adamo and C. K. Anders, "Stratifying Triple-Negative Breast Cancer: Which Definition(s) to Use?” Breast Cancer Research, Vol. 13, No. 2, 2011, p. 105. doi:10.1186/bcr2852

[13] K. P. Siziopikou and M. Cobleigh, "The Basal Subtype of Breast Carcinomas May Represent the Group of Breast Tumors That Could Benefit from EGFR-Targeted Therapies,” Breast, Vol. 16, No. 1, 2007, pp. 104-107. doi:10.1016/j.breast.2006.09.003

[14] K. P. Siziopikou, D. C. Gingell, K. Proussaloglou, et al., "Basal Subtype and EGFR Expression in Breast Carcinomas, Evidence of Racial Differences,” Breast Cancer Research and Treatment, Vol. 100, Supp. 1, 2006, p. 141

[15] L. A. Carey, C. M. Perou, C. A. Livasy, et al., "Race, Breast Cancer Subtypes, and Survival in the Carolina Breast Cancer Study,” Journal of the American Medical Association, Vol. 295, No. 21, 2006, pp. 2492-2502. doi:10.1001/jama.295.21.2492

[16] M. van de Rijn, C. M. Perou, R. Tibshirani, et al., "Expression of Cytokeratins 17 and 5 Identifies a Group Of Breast Carcinomas with Poor Clinical Outcome," American Journal of Pathology, Vol. 161, No. 6, 2002, pp. 1991-1996. doi:10.1016/S0002-9440(10)64476-8

[17] K. F. Trivers, M. J. Lund, P. L. Porter, et al., "The Epidemiology of Triple-Negative Breast Cancer, including Race,” Cancer Causes \& Control, Vol. 20, No. 7, 2009, pp. 1071-1082. doi:10.1007/s10552-009-9331-1

[18] R. C. Millikan, B. Newman, C. K. Tse, et al., "Epidemiology of Basal-Like Breast Cancer," Breast Cancer Research and Treatment, Vol. 109, No. 1, 2008, pp. 123139. doi:10.1007/s10549-007-9632-6
[19] J. D. Albano, E. Ward, A. Jemal, et al., "Cancer Mortality in the United States by Education Level and Race," Journal of the National Cancer Institute, Vol. 99, No. 18, 2007, pp. 1384-1394. doi:10.1093/jnci/djm127

[20] N. N. A. Howlader, M. Krapcho, N. Neyman, R. Aminou, W. Waldron, S. F. Altekruse, C. L. Kosary, J. Ruhl, Z. Tatalovich, H. Cho, A. Mariotto, M. P. Eisner, D. R. Lewis, H. S. Chen, E. J. Feuer, K. A. Cronin and B. K. Edwards, "SEER Cancer Statistics Review, 1975-2008," National Cancer Institute, Bethesda, Based on November 2010 Seasonal Energy Efficiency Ratio Data Submission.

[21] K. S. Albain, J. M. Unger, J. J. Crowley, C. A. Coltman Jr. and D. L. Hershman, "Racial Disparities in Cancer Survival among Randomized Clinical Trials Patients of the Southwest Oncology Group,” Journal of the National Cancer Institute, Vol. 101, No. 14, 2009, pp. 984-992. doi:10.1093/jnci/djp175

[22] C. K. Anders, D. S. Hsu, G. Broadwater, et al., "Young Age at Diagnosis Correlates with Worse Prognosis and Defines a Subset of Breast Cancers with Shared Patterns of Gene Expression,” Journal of Clinical Oncology, Vol. 26, No. 20, 2008, pp. 3324-3330. doi:10.1200/JCO.2007.14.2471

[23] American Cancer Society, "Breast Cancer Facts \& Figures 2009-2010,” American Cancer Society, Inc., Atlanta, 2009-2010.

[24] N. Klauber-DeMore, “Tumor Biology of Breast Cancer in Young Women,” Breast Diseases, Vol. 23, 2005, pp. 915.

[25] K. Oshima, Y. Naoi, K. Kishi, et al., "Gene Expression Signature of TP53 but Not Its Mutation Status Predicts Response to Sequential Paclitaxel and 5-FU/Epirubicin/Cyclophosphamide in Human Breast Cancer," Cancer Letters, Vol. 307, No. 2, 2011, pp. 149-157. doi:10.1016/j.canlet.2011.03.027

[26] P. D. Pharoah, N. E. Day and C. Caldas, "Somatic Mutations in the p53 Gene and Prognosis in Breast Cancer: A Meta-Analysis,” British Journal of Cancer, Vol. 80, No. 12, 1999, pp. 1968-1973. doi:10.1038/sj.bjc.6690628

[27] L. D. Miller, J. Smeds, J. George, et al., “An Expression Signature for p53 Status in Human Breast Cancer Predicts Mutation Status, Transcriptional Effects, and Patient Survival," Proceedings of the National Academy of Sciences of the United States of America, Vol. 102, No. 38, 2005, pp. 13550-13555. doi:10.1073/pnas.0506230102

[28] B. T. Hennessy, A. M. Gonzalez-Angulo, K. StemkeHale, et al., "Characterization of a Naturally Occurring Breast Cancer Subset Enriched in Epithelial-to-Mesenchymal Transition and Stem Cell Characteristics," Cancer Research, Vol. 69, No. 10, 2009, pp. 4116-4124. doi:10.1158/0008-5472.CAN-08-3441

[29] Z. Jiang, T. Deng, R. Jones, et al., "Rb Deletion in Mouse Mammary Progenitors Induces Luminal-B or Basal-Like/ EMT Tumor Subtypes Depending on p53 Status,” Journal of Clinical Investigation, Vol. 120, No. 9, 2010, pp. 3296-3309. doi:10.1172/JCI41490 


\section{Abbreviations}

AA: African American; TNBC: Triple Negative Breast Carcinoma; CK 5/6: Cytokeratin 5/6; LVI: Lymphatic Vascular Invasion; ER: Estrogen Receptor; PR: Pro-
gester-One Receptor; HER2: Epidermal Growth Factor Receptor; PARP-1 Inhibitors: Poly-(ADP-Ribose) Polymerases 1 Inhibitors; TMA: Tissue Microarray. 